\title{
Backflow and Density Excitations in Quantum Fluids
}

\author{
Victor K. Wong* \\ Department of Physics, University of Michigan, Ann Arbor, Michigan
}

(Received June 10, 1974)

By the use of the f sum rule and a separation of density excitations into single and multiple excitations, we derive exact quantum expressions for the backflow contribution to the dynamic structure function $\mathrm{S}(\mathbf{k}, \omega)$ and for the discrete single-excitation spectrum $\omega_{\mathrm{k}}$. The derivation is carried out for Bose liquids, Fermi liquids, charged quantum liquids, quantum solutions, an impurity atom dissolved in quantum fluids, and phonons in solids. It is shown that the backflow arises from virtual multiexcitations and that real multiexcitations give rise to a background. It is argued that multiexcitations are relatively insensitive to long-range order or quantum statistics. Thus the backflow and background contributions to $\mathbf{S}(\mathbf{k}, \omega)$ of the liquid and solid phases of ${ }^{4} \mathrm{He}$, and also that to $\mathbf{S}(\mathbf{k})$ of liquid ${ }^{3} \mathrm{He}$ and of liquid ${ }^{4} \mathrm{He}$, are expected to be similar, which is consistent with existing data. The exact expression for $\omega_{\mathrm{k}}$, which shows that multiexcitations effectively repel the single excitations, is used to make some speculations concerning the large-wavevector phonon spectrum in liquid ${ }^{3} \mathrm{He}$ and in ${ }^{3} \mathrm{He}-{ }^{4} \mathrm{He}$ solutions.

\section{INTRODUCTION}

Quantum fluids are usually classified in terms of the quantum statistics of the particles and the nature (gap, slope, etc.) of the quasiparticle spectrum. The usual scattering experiments, however, do not measure directly the quasiparticle spectrum but rather the excitation spectrum of density fluctuations. Fortunately, density excitations in quantum fluids are amenable to a powerful and general theory involving the dynamic structure function (see, e.g., Ref. 1) and its related sum rules. The purpose of this paper is to add, and to illustrate the use of, one more piece of equipment to this powerful machinery, viz., exact quantum expressions for the backflow and the discrete density excitation spectrum.

\footnotetext{
*Supported in part by a Faculty Research Grant from the Horace H. Rackham School of Graduate Studies at the University of Michigan.
} 
The dynamic structure function $S(\mathbf{k}, \omega)$, which contains the maximum information that can be obtained about a quantum fluid in linear response to a density probe, can be defined at zero temperature as

$$
S(\mathbf{k}, \omega)=\sum_{v}\left|\left(\rho_{\mathbf{k}}^{+}\right)_{v 0}\right|^{2} \delta\left(\omega-\omega_{v 0}\right)
$$

The summation is over the complete set of exact energy eigenstates $|v\rangle$ with total momentum $\mathbf{k} ;|v\rangle$ is coupled to the exact ground state $|0\rangle$ by density fluctuations $\rho_{\mathbf{k}}^{+}$with matrix elements $\left(\rho_{\mathbf{k}}^{+}\right)_{v 0}$; and $\omega_{v 0} \equiv \omega_{v}-\omega_{0}$ is the exact excitation frequency. To be specific, we consider as a prototype the Bose liquid; other examples abound in the literature and will be treated later. If $\rho_{\mathbf{k}}^{+}$couples $|0\rangle$ only to the one-phonon state $|1\rangle$ and to no other, one finds with the aid of the $f$ sum rule the well-known Feynman ${ }^{2}$ one-phonon excitation spectrum,

$$
\omega_{k}^{\mathrm{F}}=k^{2} / 2 m S(\mathbf{k})
$$

where $S(\mathbf{k})$ is the static structure function. Since (2) overestimated the excitation spectrum of liquid ${ }^{4} \mathrm{He}$, Feynman and $\mathrm{Cohen}^{3}$ (FC) attempted to lower the spectrum by introducing the concept of backflow on the basis of semiclassical and physical arguments involving number conservation. Subsequently Miller, Pines, and Nozieres ${ }^{4}$ (MPN) advanced a microscopic picture of backflow in terms of an excitation dragging a cloud of virtual excitations which would serve to reduce the excitation energy as well as to conserve number. In both of these approaches, the physical picture of backflow is developed in terms of the motion of an impurity atom through the Bose liquid. To gain an understanding of backflow in a pure quantum liquid, one had to proceed by analogy or from a picturesque and qualitative description.

An offshoot of the vagueness connected with backflow can be found in the widely used ${ }^{4-8}$ two-term separation of $S(\mathbf{k}, \omega)$ of liquid ${ }^{4} \mathrm{He}$

$$
N^{-1} S(\mathbf{k}, \omega)=Z(\mathbf{k}) \delta\left(\omega-\omega_{k}\right)+X(\mathbf{k}, \omega)
$$

Equation (3) was first written down by $\mathrm{MPN},{ }^{4}$ who interpreted the $\delta$ function term as the discrete contribution coming from "single-particle excitation from the condensate" and the second term $X(\mathbf{k}, \omega)$ as the continuum contribution coming from "multi-particle excitations." Such an interpretation is motivated by the fact that if $\rho_{\mathbf{k}}^{+}$couples $|0\rangle$ only to the one-phonon state $|1\rangle$, then the background $X(\mathbf{k}, \omega)$ disappears and only the $\delta$-function term remains. The remaining one-phonon state is identical to the Feynman one-phonon state which contains no backflow. However, it is not clear whether the introduction of backflow would change the MPN interpretation or even the form of (3). 
In this paper, which is largely pedagogical, we shall be concerned with making more precise the vague but physically obvious notion of backflow from a purely quantum mechanical point of view, i.e., beginning with Eq. (1). Although the basic idea of this paper is extremely simple, we feel that the implications of this simple idea are sufficiently important to warrant a full discussion. In Section 2 we divide the density excitations into two groups: the single excitations and the multiexcitations. This division and the $f$ sum rule are used to derive exact expressions for the backflow contribution to $S(\mathbf{k}, \omega)$ and for the discrete single-excitation spectrum. It is shown that the backflow arises from virtual multiexcitations and the real multiexcitations give rise to a background. The derivation is carried out for a variety of quantum fluids: Bose liquids, Fermi liquids, charged quantum liquids, quantum solutions, an impurity atom dissolved in quantum fluids, and phonons in solids. It is shown that, contrary to the MPN interpretation of (3), $S(\mathbf{k}, \omega)$ can be separated into three contributions (single-excitation, backflow, and background) such that the residue $Z(\mathbf{k})$ in (3) contains in fact multiexcitation contributions through the backflow. In a neutral fluid the backflow contribution to $S(\mathbf{k})$ is shown to appear first at the $k^{3}$ term, whereas the background contribution is shown to contribute to the $k^{4}$ term. On the other hand, in a charged fluid, both the backflow and background contributions to $S(\mathbf{k})$ are shown to be order $k^{4}$. Thus, the Feynman relation (2) is shown to break down at a term $k^{2}$ higher than the leading order, because of backflow for neutral fluids (backflow and background for charged fluids). The exact expression for the discrete density excitation spectrum $\omega_{k}$ emphasizes the role of multiexcitations (backflow and background) in lowering the Feynman spectrum $\omega_{k}^{F}$ to the exact $\omega_{k}$. It is shown that the effective mass $m_{\mathrm{i}}^{*}$ of an impurity atom of mass $m_{\mathrm{i}}$ dissolved in a quantum fluid is generated solely by the backflow and that $m_{\mathrm{i}}^{*} \geq m_{\mathrm{i}}$. For a system of phonons in a solid, it is emphasized that the Debye-Waller factor plays the role of the elastic form factor and is not in general the intensity function of the one-phonon $\delta$-function.

In Section 3 we discuss some specific applications of the exact results. It is argued that the backflow and background contributions to $S(\mathbf{k}, \omega)$ are insensitive to the presence or absence of long-range order. Existing data on $S(\mathbf{k}, \omega)$ in the liquid and solid phases of ${ }^{4} \mathrm{He}$ appear to confirm these expectations for both the backflow and background. It is pointed out that the role of Debye-Waller factor $e^{-2 W}$ in a solid is similar to that of the condensate density $n_{0}$ in a Bose liquid. In neutral quantum fluids, the interplay of the negative backflow contribution and the positive background contribution is shown to lead to an inflection point in $S(\mathbf{k})$ at small $k$, independent of quantum statistics. Estimates of these inflection points in liquid ${ }^{4} \mathrm{He}$ and in liquid ${ }^{3} \mathrm{He}$ are consistent with experiments. Finally, 
speculations concerning the shape of the phonon spectrum at large wave vectors in liquid ${ }^{3} \mathrm{He}$ and in ${ }^{3} \mathrm{He}-{ }^{4} \mathrm{He}$ solutions are presented.

\section{BACKFLOW AND DENSITY EXCITATIONS}

The concept of backflow was introduced by $\mathrm{FC}^{3}$ and by $\mathrm{MPN}^{4}$ on the basis of an application of number conservation. It is well known that the $f$ sum rule is a direct consequence of number conservation, barring velocity-dependent interparticle potentials. To derive an exact quantum expression for backflow, it is clear that we need to exploit the $f$ sum rule:

$$
\sum_{v}\left|\left(\rho_{\mathbf{k}}^{+}\right)_{\nu 0}\right|^{2} \omega_{\nu 0}=N k^{2} / 2 m
$$

where $N$ is the total number of bosons (fermions) and $m$ is the mass of the boson (fermion). The general approach is to separate the exact eigenstates $|v\rangle$ into the single-excitation states $|1\rangle$ and the multiexcitation states $|n\rangle$, where the single-excitation states $|1\rangle$ are defined as those states that would exhaust the $f$ sum rule in the long-wavelength limit. In other words, the states $|\mathbf{1}\rangle$, which may have a discrete and/or continuous spectrum, satisfy

$$
\lim _{k \rightarrow 0}\left|\left(\rho_{\mathbf{k}}\right)_{10}^{+}\right|^{2} \omega_{10}=N k^{2} / 2 m
$$

where the sum over the continuous parts of $\omega_{10}$ is understood. It seems reasonable to assume that the separation of $|v\rangle$ into $|1\rangle$ and $|n\rangle$, although defined only in the $k \rightarrow 0$ limit, remains valid over a finite range in $k$ as long as the states $|1\rangle$ are well defined. Effecting such a separation in (4) and substituting back in (1) yields the desired expression for the backflow contribution to $S(\mathbf{k}, \omega)$.

\subsection{Bose Liquids}

We apply this approach first to Bose liquids, and make no assumption about the presence or absence of Bose-Einstein condensation. It is a wellknown fact that in the $k \rightarrow 0$ limit the phonon exhausts the $f$ sum rule. The excited states $|v\rangle$ can thus be divided into $|1\rangle$, the one-phonon state, and the remaining state $|n\rangle$, the multiphonon states. Because of phonon-phonon interaction, the energy eigenstate $|1\rangle$ cannot have a definite number of (bare) phonons; however, the renormalized (experimental) one-phonon state $^{7}$ is well defined and can be approximated by a discrete energy eigenstate over a wide range of $k$. It is in the latter spirit that we call the energy eigenstate $|1\rangle$ a discrete one-phonon state and ignore the width of the state. Separating out the one-phonon state in (4), we find

$$
\left|\left(\rho_{k}^{+}\right)_{10}\right|^{2}=N\left(k^{2} / 2 m \omega_{k}\right)-\omega_{k}^{-1} \sum_{n}\left|\left(\rho_{k}^{+}\right)_{n 0}\right|^{2} \omega_{n 0}
$$


where $\omega_{k} \equiv \omega_{10}$ is the discrete one-phonon spectrum. Inserting (5) into the definition (1), we find a natural three-term division of $S(\mathbf{k}, \omega)$ :

$$
N^{-1} S(\mathbf{k}, \omega)=Z^{\mathbf{F}}(\mathbf{k}) \delta\left(\omega-\omega_{k}\right)+Y(\mathbf{k}) \delta\left(\omega-\omega_{k}\right)+X(\mathbf{k}, \omega)
$$

where

$$
\begin{aligned}
Z^{\mathrm{F}}(\mathbf{k}) & \equiv k^{2} / 2 m \omega_{k} \\
Y(\mathbf{k}) & \equiv-\omega_{k}^{-1} N^{-1} \sum_{n}\left|\left(\rho_{k}^{+}\right)_{n 0}\right|^{2} \omega_{n 0} \leq 0 \\
X(\mathbf{k}, \omega) & \equiv N^{-1} \sum_{n}\left|\left(\rho_{k}^{+}\right)_{n 0}\right|^{2} \delta\left(\omega-\omega_{n 0}\right)
\end{aligned}
$$

The three terms on the right-hand side of (6) can now be interpreted. The first term is evidently the Feynman one-phonon contribution. The second term, $Y(\mathbf{k}) \delta\left(\omega-\omega_{k}\right)$, arises from the virtual $\left(\omega_{n 0}\right.$ need not be equal to $\left.\omega\right)$ excitation of multiphonons, as can be seen from (8). In contrast, the last term $X(\mathbf{k}, \omega)$ [see (9)] arises from excitation of real $\left(\omega_{n 0}\right.$ must be equal to $\left.\omega\right)$ multiphonons. In keeping with the usual microscopic picture of backflow in terms of virtual excitations, we can identify (8) as the exact quantum expression for the backflow contribution to $S(\mathbf{k}, \omega)$. Furthermore, (9) can now be called the background contribution. Comparing (6) with (3), we see that the residue $Z(\mathbf{k})$ in (3) cannot be interpreted as arising solely from one-phonon contributions but contains multiphonon contributions through the backflow term, $Z(\mathbf{k})=Z^{\mathrm{F}}(\mathbf{k})+Y(\mathbf{k})$.

In the $k \rightarrow 0$ limit, we know by construction that only the one-phonon term $Z^{\mathrm{F}}(\mathbf{k}) \delta\left(\omega-\omega_{k}\right)$ survives. Hence the three-term division (6-9) of $S(\mathbf{k}, \omega)$ makes sense in the small- $k$ limit only if the backflow and background contributions are relatively small and can be considered as corrections to the one-phonon contribution. To demonstrate this, we define the structure functions

$$
N S_{l}(\mathbf{k})=\int_{0}^{\infty} d^{l} \omega \omega^{l} S(\mathbf{k}, \omega), \quad l=-1,0,1,2, \ldots
$$

where $S_{0}(\mathbf{k}) \equiv S(\mathbf{k})$ is the static structure function. Inserting (6) into (10), we find

$$
\begin{gathered}
S_{l}(\mathbf{k})=Z^{\mathrm{F}}(\mathbf{k}) \omega_{k}^{l}+Y(\mathbf{k}) \omega_{k}^{l}+X_{l}(\mathbf{k}) \\
X_{l}(\mathbf{k}) \equiv N^{-1} \sum_{n}\left|\left(\rho_{k}^{+}\right)_{n 0}\right|^{2}\left(\omega_{n 0}\right)^{l} \geq 0
\end{gathered}
$$

Following the standard sum rule arguments of MPN which we do not reproduce here, we conclude that in the small- $k$ limit, $\left(\rho_{k}^{+}\right)_{n 0} \propto k^{2}$ and $\omega_{n 0}$ is a constant since $|n\rangle$ is a multiphonon state. From (8) we see that $Y(k) \propto k^{3}$, 
and from (12), $X_{l}(k) \propto k^{4}$. Hence as $k \rightarrow 0$ the one-phonon Feynman result is obtained, as expected. Furthermore, we see that the Feynman relation (2) breaks down at the $k^{3}$ term because of backflow, rather than at the $k^{4}$ background term. Therefore as $k \rightarrow 0$ the correction due to backflow dominates the background contribution to $S(\mathbf{k})$.

The identification of (8) as the exact quantum expression for backflow can now be checked for the features ascribed to backflow by FC. First we consider number conservation. From (8) and (12) we see that $Y(\mathbf{k})$ satisfies

$$
Y(\mathbf{k})=-X_{1}(\mathbf{k}) / \omega_{k}
$$

From $l=1$ in (11), it is apparent that the $f$ sum is satisfied if and only if (13) is satisfied. Hence number conservation is satisfied, since $Y(\mathbf{k})$ was constructed so. A further feature ascribed to backfiow by $\mathrm{FC}$ is that the one-phonon spectrum should be lowered if backflow and the concomitant background are taken into account. Using (11) and (13), we find the following simple, exact, and equivalent expressions for $\omega_{k}$, which arise directly from the division (6):

$$
\begin{aligned}
\omega_{k} & =\left[\left(k^{2} / 2 m\right)-X_{1}(\mathbf{k})\right] /\left[S(\mathbf{k})-X_{0}(\mathbf{k})\right] \\
\omega_{k}^{2} & =\left[\left(k^{2} / 2 m\right)-X_{1}(\mathbf{k})\right] /\left[S_{-1}(\mathbf{k})-X_{-1}(\mathbf{k})\right] \\
\omega_{k} & =\left[S(\mathbf{k})-X_{0}(\mathbf{k})\right] /\left[S_{-1}(\mathbf{k})-X_{-1}(\mathbf{k})\right]
\end{aligned}
$$

It is useful to rewrite (14a) in the form

$$
\omega_{k}=\omega_{k}^{\mathrm{F}}+\left[X_{0}(\mathbf{k}) / S(\mathbf{k})\right]\left[\omega_{k}-\bar{\omega}_{n 0}\right]
$$

where $\bar{\omega}_{n 0} \equiv X_{1}(\mathbf{k}) / X_{0}(\mathbf{k})$ is the average multiexcitation energy. By definition, $\bar{\omega}_{n 0} \geq \omega_{k}$ and from the positivity of $X_{0} / S$ we find

$$
\omega_{k} \leq \omega_{k}^{\mathrm{F}}
$$

Hence the Feynman spectrum $\omega_{k}^{\mathrm{F}}$ is lowered because of the presence of multiexcitations with an average energy higher than $\omega_{k}$. Loosely speaking, the multiexcitations repel the spectrum $\omega_{k}^{\mathrm{F}}$ to a lower energy $\omega_{k}$.

The three-term division (6)-(9) was first derived in a rigorous microscopic calculation of $S\left(\mathbf{k}_{,} \omega\right)$ in the long-wavelength limit in a simple one-parameter model of a Bose gas. ${ }^{9,10}$ The explicit expansions in $k$ of the functions $Z^{\mathrm{F}}(\mathbf{k})$, $Y(\mathbf{k})$, and $X_{i}(\mathbf{k})$ in Refs. 9 and 10 can be used to verify the above results. All expressions are verified with one exception: $X_{-1}(\mathbf{k})$ was found in the rigorous model calculation to be $\propto k^{4} \ln k$. Hence the simple sum rule arguments are not precise enough to give logarithmic dependences. On the other hand, since in the rigorous model calculation we found $Y(\mathbf{k}) \propto k^{3}$ with no logarithmic factor, we have no reason to doubt the sum rule arguments for the small- $k$ dependence of the backflow $Y(\mathbf{k})$. 
We emphasize that the $f$ sum rule, rather than another $\omega^{l}$ sum rule, is used here to determine the backflow. Other $\omega^{l}$ sum rules involve new functions and thus are not suitable. The exception is the $\omega^{\circ}$ sum rule, which is really not a sum rule but a definition of $S(\mathbf{k})$; thus it does not act as a physical constraint, in contrast to the $f$ sum rule. The separation of $S(\mathbf{k}, \omega)$ effected by Hall and Feenberg ${ }^{6}$ can be viewed as one based on the $\omega^{0}$ sum rule.

\subsection{Fermi Liquids}

We consider here normal Fermi liquids with couplings sufficiently strong that the zero-sound mode is well defined. For such Fermi liquids, the single-excitation states consist of a discrete zero-sound state $|1\rangle$ and a continuous single-pair state $|2\rangle$. Since $\omega_{k} \equiv \omega_{10}$ is discrete, we separate out the $|1\rangle$ state in the $f$ sum rule (4) to get

$$
\begin{aligned}
\left|\left(\rho_{k}^{+}\right)_{10}\right|^{2}= & N\left(k^{2} / 2 m \omega_{k}\right)-\omega_{k}^{-1} \sum_{\mathbf{q}}\left|\left(\rho_{k}^{+}\right)_{20}\right|^{2} \omega_{\mathbf{q} \mathbf{k}} \\
& -\omega_{k}^{-1} \sum_{n}\left|\left(\rho_{k}^{+}\right)_{n 0}\right|^{2} \omega_{n 0}
\end{aligned}
$$

where $\omega_{\mathbf{q} \mathbf{k}} \equiv \omega_{20}$ is the continuous single-pair spectrum that depends on the intermediate momentum q. Substituting (17) into (1), we find

$$
N^{-1} S(\mathbf{k}, \omega)=N^{-1} S^{\mathrm{L}}(\mathbf{k}, \omega)+Y(\mathbf{k}) \delta\left(\omega-\omega_{k}\right)+X(\mathbf{k}, \omega)
$$

where the single-excitation contributions are grouped together into the Landau dynamic structure function

$$
\begin{aligned}
N^{-1} S^{\mathrm{L}}(\mathbf{k}, \omega) & =\left[\left(k^{2} / 2 m \omega_{k}\right)+Y^{\mathrm{P}}(\mathbf{k})\right] \delta\left(\omega-\omega_{k}\right)+X^{\mathrm{P}}(\mathbf{k}, \omega) \\
Y^{\mathrm{P}}(\mathbf{k}) & \equiv-\omega_{k}^{-1} N^{-1} \sum_{\mathbf{q}}\left|\left(\rho_{k}^{+}\right)_{20}\right|^{2} \omega_{\mathbf{q} \mathbf{k}} \\
X^{\mathbf{P}}(\mathbf{k}, \omega) & \equiv N^{-1} \sum_{\mathbf{q}}\left|\left(\rho_{k}^{+}\right)_{20}\right|^{2} \delta\left(\omega-\omega_{\mathbf{q} \mathbf{k}}\right)
\end{aligned}
$$

The multiexcitation functions $Y(\mathbf{k})$ and $X(\mathbf{k}, \omega)$ are given formally by the same expressions as in Eqs. (8)-(9). As before, we introduce the structure functions (10), which can be written

$$
S_{l}(\mathbf{k})=S_{l}^{\mathrm{L}}(\mathbf{k})+Y(\mathbf{k}) \omega_{k}^{l}+X_{l}(\mathbf{k})
$$

where

$$
\begin{aligned}
S_{l}^{\mathrm{L}}(\mathbf{k}) & =\left[Z^{\mathrm{F}}(\mathbf{k})+Y^{\mathrm{P}}(\mathbf{k})\right] \omega_{k}^{l}+X_{l}^{\mathrm{P}}(\mathbf{k}) \\
X_{l}^{\mathrm{P}}(\mathbf{k}) & \equiv N^{-1} \sum_{\mathbf{q}}\left|\left(\rho_{k}^{+}\right)_{20}\right|^{2}\left(\omega_{\mathbf{q k}}\right)^{l}
\end{aligned}
$$


Only processes involving single excitations are included in the Landau theory; those involving multiexcitations are completely ignored. In particular the backflow of virtual single pairs are included; and from (20) and (24) we see that the single-pair backflow $Y^{\mathrm{P}}(\mathbf{k})$ satisfies $Y^{\mathrm{P}}(\mathbf{k})=-X_{1}^{\mathrm{P}}(\mathbf{k}) / \omega_{k}$, which ensures that $S^{\mathrm{L}}(\mathbf{k}, \omega)$ satisfies the $f$ sum rule. The corrections to the Landau theory are contained in the two multiexcitation contributions, backflow $Y(\mathbf{k})$ and background $X(\mathbf{k}, \omega)$, which are formally given by the same expressions as those for the Bose liquid. Hereafter the word backflow (without any adjectives) shall be used to refer to this universal backflow due to virtual multiexcitations.

To obtain the small- $k$ dependences of the various terms in (22)-(24), we use the standard arguments as found in Ref. 1 to estimate the matrix elements $\left(\rho_{k}^{+}\right)_{n 0}$. We find that as $k \rightarrow 0, Y^{\mathrm{P}}(\mathbf{k}) \propto k, X_{l}^{\mathrm{P}}(\mathbf{k}) \propto k^{1+l}, S_{l}^{\mathrm{L}}(\mathbf{k})$ $\propto k^{1+l}, Y(\mathbf{k}) \propto k^{3}$, and $X_{l}(\mathbf{k}) \propto k^{4}$. From the compressibility sum rule $(l=-1)$, we see that the difference between the (first) sound speed $c_{1}$ and the zero-sound speed $c_{0}=\lim _{k \rightarrow 0}\left(\omega_{k} / k\right)$ arises solely from the single-pair states, which justifies the calculation of the sound speed difference ${ }^{1}$ within Landau's theory. This fact is well known; the present emphasis is that backflow does not alter the statement. From the $\omega^{0}$ sum rule $(l=0)$, we see that because of backflow the Landau expression for $S(\mathbf{k})$ breaks down at the $k^{3}$ term, rather than the $k^{4}$ background term. Once again as $k \rightarrow 0$ the backflow contribution dominates background contribution.

As in the derivation of (14), the discrete zero-sound spectrum $\omega_{k}$ can be written

$$
\omega_{k}=\frac{\left(k^{2} / 2 m\right)-X_{1}^{\mathrm{P}}(\mathbf{k})-X_{1}(\mathbf{k})}{S(\mathbf{k})-X_{0}^{\mathrm{P}}(\mathbf{k})-X_{0}(\mathbf{k})}
$$

The zero-sound spectrum in Landau's theory $\omega_{k}^{\mathrm{L}}$ corresponds to neglecting $X_{1}(\mathbf{k})$ and $X_{0}(\mathbf{k})$ in $(25)$ and can be considered the Fermi analog of the Feynman spectrum $\omega_{k}^{\mathrm{F}}$. Equation (25) can be rewritten many ways, in particular

$$
\omega_{k}=\omega_{k}^{\mathrm{L}}+\frac{X_{0}(\mathbf{k})}{S(\mathbf{k})-X_{0}^{\mathrm{P}}(\mathbf{k})}\left[\omega_{k}-\bar{\omega}_{n 0}\right]
$$

where $\bar{\omega}_{n 0}$ is the average multiexcitation energy. Since $S(\mathbf{k})-X_{0}^{\mathrm{P}}(\mathbf{k})$ is positive, the sign of the shift $\omega_{k}-\omega_{k}^{\mathrm{L}}$ depends on the sign of $\omega_{k}-\bar{\omega}_{n 0}$. Hence, roughly speaking, the multiexcitations can be thought of as repelling the zero-sound mode from $\omega_{k}^{\mathrm{L}}$ to $\omega_{k}$.

\subsection{Charged Quantum Fluids}

The discussions in Sections 2.1 and 2.2 have been restricted to neutral quantum fluids in which the two-particle interaction is short range. Because 
of the long-range Coulomb potential in a charged fluid, the discrete phonon mode found in the neutral fluids is drastically changed to the discrete plasmon mode. The major distinction, however, is that in a charged quantum fluid the plasmon state $|1\rangle$ by itself exhausts the $f$ sum rule as $k \rightarrow 0$. Hence the multiexcitation state $|n\rangle$ includes the single-pair state. The resulting $S(\mathbf{k}, \omega)$ has the same form as (6)-(9), where $\omega_{k}$ is to be interpreted as the plasmon mode. Likewise, the structure functions are given by (11)-(12). Since $\omega_{k}$ $\rightarrow$ const as $k \rightarrow 0$, we have $Y(\mathbf{k}) \propto k^{4}, X_{l}(\mathbf{k}) \propto k^{4}$ as $k \rightarrow 0$. Hence for a charged quantum fluid, the Feynman relation breaks down at the $k^{4}$ term, where both backflow and background contribute. In this sense, at long wavelengths backflow is not as important for a charged fluid as for the neutral fluid. Such a conclusion is also apparent from comparing the discrete single-excitation spectrum given by (14) and (25).

\subsection{Quantum Solutions}

We consider a two-component quantum solution which is a mixture of a Bose liquid and a Fermi liquid. More components with either statistics can be easily handled if necessary. To be specific, we consider neutral components. The dynamic structure function $S(\mathbf{k}, \omega)$ for the system is still defined as in (1) but with

$$
\rho_{k}=\rho_{k}^{\mathrm{b}}+\rho_{k}^{\mathrm{f}}
$$

where $\rho_{k}^{\mathrm{b}}$ and $\rho_{k}^{\mathrm{f}}$ are density fluctuations for the bosons and fermions. The $f$ sum rule takes on a slight modification:

$$
\sum_{v}\left|\left(\rho_{k}^{+}\right)_{v 0}\right|^{2} \omega_{v 0}=N k^{2} / 2 \mu
$$

where $N=N_{\mathrm{b}}+N_{\mathrm{f}}$ is the total number of bosons and fermions, the reduced mass $\mu$ is defined by

$$
\frac{1}{\mu} \equiv \frac{N_{\mathrm{b}}}{N m_{\mathrm{b}}}+\frac{N_{\mathrm{f}}}{N m_{\mathrm{f}}}
$$

and $m_{\mathrm{b}}\left(m_{\mathrm{f}}\right)$ is the mass of the bosons (fermions). Equation (28) together with (27) and (29) is implied by the following partial $f$ sum rule:

$$
\begin{aligned}
\sum_{v}\left|\left(\rho_{k}^{+}\right)_{v 0}^{\alpha \beta}\right|^{2} \omega_{v 0} & =\left(N_{\alpha} N_{\beta}\right)^{1 / 2}\left(k^{2} / 2 m_{\alpha}\right) \delta_{\alpha \beta}, \quad \alpha, \beta=\mathrm{b}, \mathrm{f} \\
2\left|\left(\rho_{k}^{+}\right)_{v 0}^{\alpha \beta}\right|^{2} & \equiv\left\langle 0\left|\rho_{k}^{\alpha}\right| v\right\rangle\left\langle v\left|\rho_{k}^{\beta+}\right| 0\right\rangle+\left\langle 0\left|\rho_{k}^{\beta}\right| v\right\rangle\left\langle v\left|\rho_{k}^{\alpha+}\right| 0\right\rangle
\end{aligned}
$$

The single-excitation states consist of the phonon state $|1\rangle$ and the singlepair state $|2\rangle$. The discrete phonon state $|1\rangle$ is coupled to the ground state $|0\rangle$ by any density fluctuation $\rho_{k}^{\alpha+}, \alpha=\mathrm{b}$ or $\mathrm{f}$, whereas the continuous 
single-pair state $|2\rangle$ is coupled to $|0\rangle$ only by $\rho_{k}^{\mathrm{f}+}$. Separating the discrete phonon state $|1\rangle$ in (28), we get

$\left|\left(\rho_{k}^{+}\right)_{10}\right|^{2}=N\left(k^{2} / 2 \mu \omega_{k}\right)-\omega_{k}^{-1} \sum_{\mathbf{q}}\left|\left(\rho_{k}^{\mathrm{f}}+\right)_{20}\right|^{2} \omega_{\mathbf{q k}}-\omega_{k}^{-1} \sum_{n}\left|\left(\rho_{k}^{+}\right)_{n 0}\right|^{2} \omega_{n 0}$

Substituting (31) into (1), we get again a three-term division of $S(\mathbf{k}, \omega)$ :

$$
N^{-1} S(\mathbf{k}, \omega)=N^{-1} S^{\mathrm{S}}(\mathbf{k}, \omega)+Y(\mathbf{k}) \delta\left(\omega-\omega_{k}\right)+X(\mathbf{k}, \omega)
$$

where the single excitation $S^{\mathrm{s}}(\mathbf{k}, \omega)$ is given by

$$
N^{-1} S^{\mathrm{S}}(\mathbf{k}, \omega)=\left[k^{2} / 2 \mu \omega_{k}+Y^{\mathbf{P}}(\mathbf{k})\right] \delta\left(\omega-\omega_{h}\right)+X^{\mathrm{P}}(\mathbf{k}, \omega)
$$

The single-pair functions $Y^{\mathrm{P}}(\mathbf{k})$ and $X^{\mathrm{P}}(\mathbf{k}, \omega)$ are given by (20) and (21) with $\rho_{k}^{+}$replaced by $\rho_{k}^{\mathrm{f}}$, and the multiexcitation functions $Y(\mathbf{k})$ and $X(\mathbf{k}, \omega)$, are given by (8) and (9) with $\rho_{k}^{+}$given by (27). Once again we see that the backflow $Y(\mathbf{k})$ is given by the same expression (8). The analysis for the structure functions (10) is similar to that in the previous sections and will not be repeated. The main point here is that the analysis of quantum solutions is essentially no different from that of a single-component system.

\subsection{Impurity Atom Dissolved in Quantum Fluids}

As mentioned in the introduction, FC and MPN developed the picture of backflow in a pure Bose liquid largely by analyzing the motion of an impurity atom through the liquid. Our present task is just the opposite: having analyzed backflow for quantum fluids, we now specialize to the impurity problem.

The system of a quantum fluid plus an impurity atom of mass $m_{\mathrm{i}}$ can be treated as a limit quantum solution in which the number of one component, the impurity component, approaches one. From (30) we see that the $f$ sum rule for impurity density fluctuations is

$$
\sum_{v}\left|\left(\rho_{k}^{i+}\right)_{v 0}\right|^{2} \omega_{v 0}=k^{2} / 2 m_{\mathrm{i}}
$$

Since $|v\rangle$ are exact eigenstates of the system with total momentum $\mathbf{k}$, there exist states $|\mathbf{k}\rangle$ in which $\mathbf{k}$ is carried entirely by the impurity atom with no (real) excitations in the fluid. Such a zero-excitation state $|\mathbf{k}\rangle$ clearly has a discrete spectrum $\varepsilon_{k} \equiv \omega_{k 0}$. Further excitation of the system must involve the fluid. For example, there can be a single excitation of the fluid with momentum $\mathbf{q}$ and the impurity with $\mathbf{k}-\mathbf{q}$; such a state $|1\rangle$ has a continuous spectrum $\omega_{10}$ that depends on q. States in which the fluid has multiexcitations are represented by $|n\rangle$. Separating out the discrete zero-excitation state $|\mathbf{k}\rangle$ in (34), we find

$$
\left|\left(\rho_{k}^{\mathrm{i}+}\right)_{k 0}\right|^{2}=\left(k^{2} / 2 m_{\mathrm{i}} \varepsilon_{k}\right)-\varepsilon_{k}^{-1} \sum_{v}^{\prime}\left|\left(\rho_{k}^{\mathrm{i}+}\right)_{v 0}\right|^{2} \omega_{v 0}
$$


where the prime denotes the absence of the state $|\mathbf{k}\rangle$ in the sum. Comparing (34) with (4) and (35) with (5), we see that Section 2.1 can be transcribed to the impurity problem by setting $\rho_{k}^{+} \rightarrow \rho_{k}^{\mathrm{i}+}, N \rightarrow 1, \omega_{k} \rightarrow \varepsilon_{k}, \sum_{n} \rightarrow \sum_{v}^{\prime}$. In particular the expressions for the impurity dynamic structure function $S(\mathbf{k}, \omega)$ can be written in analogy to (6)-(9). However, as the impurity moves through the quantum fluid, there exist two types of backflow: one consisting of virtual single excitations and the other of multiexcitations.

To find the effects of both types of backflow on the impurity, we consider the impurity static structure function $S^{\mathrm{i}}(\mathbf{k}, \omega)$, which is given by expressions analogous to (11)-(12). However, since the impurity density fluctuation is $\rho_{k}^{\mathrm{i}+}=\exp \left(i \mathbf{k} \cdot \mathbf{R}_{\mathrm{i}}\right)$, where $\mathbf{R}_{\mathbf{i}}$ is the impurity position, we find the following form-factor sum rule

$$
S^{\mathrm{i}}(\mathbf{k}) \equiv \sum_{\nu}\left|\left(\rho_{k}^{\mathrm{i}+}\right)_{\nu 0}\right|^{2}=\left\langle 0\left|\rho_{k}^{\mathrm{i}} \rho_{k}^{\mathrm{i}+}\right| 0\right\rangle=1
$$

Hence the impurity spectrum $\varepsilon_{k}$ is given by

$$
\varepsilon_{k}=\left[\left(k^{2} / 2 m_{\mathrm{i}}\right)-X_{1}^{\mathrm{i}}(\mathbf{k})\right] /\left[1-X_{0}^{\mathrm{i}}(\mathbf{k})\right]
$$

As $k \rightarrow 0,\left|\left(\rho_{k}^{\mathbf{i}+}\right)_{v o}\right|^{2} \sim k^{2}$ since the impurity current is not conserved. Since $\omega_{10}=\omega_{q}+\varepsilon_{\mathbf{k}-\mathbf{q}}, \omega_{10} \sim k^{0}$ as $k \rightarrow 0$; similarly $\omega_{n 0} \sim k^{0}$. Thus as $k \rightarrow 0$, $X_{1}^{\mathrm{i}}(\mathbf{k}) \sim k^{2}, X_{0}^{\mathrm{i}}(\mathbf{k}) \sim k^{2}$, and $\varepsilon_{k} \sim k^{2}$. If we write $\varepsilon_{k}=k^{2} / 2 m_{\mathrm{i}}^{*}$ as $k \rightarrow 0$, then the effective mass $m_{\mathrm{i}}^{*}$ is seen to be generated by the backflow $X_{1}^{\mathrm{i}}(\mathbf{k})$ :

$$
\frac{1}{m_{\mathrm{i}}^{*}}=\frac{1}{m_{\mathrm{i}}}-2 \lim _{k \rightarrow 0}\left[k^{-2} X_{1}^{\mathrm{i}}(\mathbf{k})\right]
$$

If only the states $|1\rangle$ were included in $X_{1}^{i}(\mathbf{k})$, then (38) would yield the Landau effective mass $m_{\mathrm{i}}^{\mathrm{L}}$, as calculated in Landau's Fermi liquid theory or quantum hydrodynamics. From the positivity of $X_{1}^{\mathrm{i}}(\mathbf{k})$, we see that both types of backflow can only increase the impurity mass:

$$
m_{\mathrm{i}}^{*} \geq m_{\mathrm{i}}^{\mathrm{L}} \geq m_{\mathrm{i}}
$$

which is a limiting case $\left(F_{1} \rightarrow 0\right.$ ) of the Leggett ${ }^{11}$ inequalities (see appendix).

\subsection{Phonons in a Solid}

In contrast to a liquid, a solid has a nonuniform ground-state expectation value of the local particle density. Here it is unimportant whether or not the solid is crystalline (long-range order) or not. What is important is that the lattice vibrations are well-defined phonons, where the lattice can be defined as the zero-phonon solid.

First we consider elastic scattering, in which no phonons are excited. The zero-phonon state $|\mathbf{k}\rangle$, which corresponds to the lattice carrying the 
total momentum $\mathbf{k}$ transferred to the system, has a discrete spectrum $\varepsilon_{k} \equiv \omega_{k 0}=k^{2} / 2 M$, where $M$ is the mass of the lattice. If the momentum $\mathbf{k}$ is applied to the $i$ th atom with mass $m$ at the lattice site $\mathbf{r}_{i}$, i.e., $\rho_{\mathbf{k}}^{\mathrm{i}+}$ $=\exp \left(i \mathbf{k} \cdot \mathbf{r}_{i}\right)$, as in the Mössbauer effect, the analysis in Section 2.5 can be taken over and the lattice recoiling spectrum $\varepsilon_{k}$ is given by (37). Clearly the backflow of virtual phonons generates the mass $M$ from $m$. In the limit of an infinitely large lattice $M \rightarrow \infty$, we find no recoil and (37) reduces to the $f$ sum rule (34) with $m_{\mathrm{j}} \rightarrow m$. Also the scattering becomes perfectly elastic

$$
S_{\mathrm{e} l}^{\mathrm{i}}(\mathbf{k}, \omega)=\{\exp [-2 W(\mathbf{k})]\} \delta(\omega)
$$

where the Debye-Waller factor $e^{-2 W}$ is just the elastic form factor $\left|\left(\rho_{\mathbf{k}}^{\mathrm{i}+}\right)_{k 0}\right|^{2}$. Since the static structure function $S^{i}(\mathbf{k})$ satisfies the form-factor sum rule (36), the Debye-Waller factor can be given by

$$
\begin{aligned}
e^{-2 W} & =1-\sum_{v}^{\prime}\left|\left(\rho_{\mathbf{k}}^{\mathrm{i}+}\right)_{v 0}\right|^{2}=1-k^{2} / 2 m \bar{\omega} \\
\bar{\omega} & \equiv \sum_{v}^{\prime}\left|\left(\rho_{\mathbf{k}}^{\mathrm{i}+}\right)_{v 0}\right|^{2} \omega_{v 0} / \sum_{v}^{\prime}\left|\left(\rho_{\mathbf{k}}^{\mathrm{i}+}\right)_{v 0}\right|^{2}
\end{aligned}
$$

where the average phonon energy $\bar{\omega}$ is introduced via (34). It is important to note that $e^{-2 W}$ is defined not by the $f$ sum rule (34), where $|\mathbf{k}\rangle$ does not even appear, but by the form-factor sum rule (36) with $m_{\mathrm{i}} \rightarrow m$. If, however, the momentum $\mathbf{k}$ is applied to the solid as a whole (as in scattering experiments) rather than to the $i$ th atom, then the elastic form factor $\left|\left(\rho_{\mathbf{k}}^{+}\right)_{k 0}\right|^{2}$ may contain, depending on the nature of the long-range order, interference (Bragg) peaks, and the Debye-Waller factor (the single-atom elastic form factor) plays the role of the envelope of the interference peaks. In either case, the Debye-Waller factor cannot be construed as backflow.

Now we consider inelastic scattering, in which phonons are excited. The single-excitation state is the one-phonon state $|1\rangle$, which has a discrete spectrum $\omega_{10} \equiv \omega_{\mathbf{k}}$, with a branch index $\lambda$ labeling the three linearly independent polarizations. To apply the $f$ sum rule (4), we expand $\rho_{\mathbf{k}}^{+}$in terms of the complete orthonormal set $\mathbf{e}_{\mathbf{k} \lambda}$ of polarization vectors

$$
\rho_{\mathbf{k}}^{+}=\sum_{\lambda} \rho_{\mathbf{k} \lambda}^{+}\left(\mathbf{k} \cdot \mathbf{e}_{\mathbf{k} \lambda}\right)
$$

Substituting (43) into (4) and separating the one-phonon state $|1\rangle$, we find $S(\mathbf{k}, \omega)$ in the form

$$
\begin{aligned}
N^{-1} S(\mathbf{k}, \omega) & =\sum_{\lambda} \frac{\left(\mathbf{k} \cdot \mathbf{e}_{\mathbf{k} \lambda}\right)^{2}}{2 m \omega_{\mathbf{k} \lambda}}\left[1-\frac{2 m X_{1 \lambda}(\mathbf{k})}{k^{2}}\right] \delta\left(\omega-\omega_{\mathbf{k} \lambda}\right)+X(\mathbf{k}, \omega) \\
X_{1 \lambda}(\mathbf{k}) & =N^{-1} \sum_{n}\left|\left(\rho_{\mathbf{k} \lambda}^{+}\right)_{n 0}\right|^{2} \omega_{n 0}
\end{aligned}
$$


and the background $X(\mathbf{k}, \omega)$ is given formally by (9). Note that the DebyeWaller factor does not appear in (44), in contrast to elastic scattering.

The harmonic one-phonon form of $S(\mathbf{k}, \omega)$ can be obtained by combining (43) with (4) and (36) to yield a sum rule, for a given $\lambda$, in the form

$$
\frac{\sum_{v}^{\prime}\left|\left(\rho_{\mathbf{k} \lambda}^{+}\right)_{v 0}\right|^{2} \omega_{v 0}}{1+e^{2 W} \sum_{v}^{\prime}\left|\left(\rho_{\mathbf{k}}^{i+}\right)_{v 0}\right|^{2}}=N \frac{\left(\mathbf{k} \cdot \mathbf{e}_{\mathbf{k} \lambda}\right)^{2}}{2 m} e^{-2 W}
$$

Expanding the denominator in (45), we find that in the one-phonon approximation the left-hand side of (45) reduces to $\left|\left(\rho_{\mathbf{k} \lambda}^{+}\right)_{10}\right|^{2} \omega_{\mathbf{k} \lambda}$, which results in the harmonic one-phonon $S^{\mathrm{H}}(\mathbf{k}, \omega)$ :

$$
N^{-1} S^{\mathrm{H}}(\mathbf{k}, \omega)=e^{-2 W(\mathbf{k})} \sum_{\lambda} \frac{\left(\mathbf{k} \cdot \mathbf{e}_{\mathbf{k} \lambda}\right)^{2}}{2 m \omega_{\mathbf{k} \lambda}} \delta\left(\omega-\omega_{\mathbf{k} \lambda}\right)
$$

Inclusion of the multiphonons neglected in (46) gives rise to two effects: First the inelastic form factors by virtue of the form-factor sum rule (36) cancel the Debye-Waller factor, and second the multiphonons by virtue of the $f$ sum rule (4) introduce backflow and background terms-the net result being that (46) turns into (44). Hence the Debye-Waller factor in general should not be confused with the intensity coefficient of the onephonon $\delta$-function.

\section{DISCUSSION}

In the present approach, a basic distinction is made between single excitations, i.e., density excitations that exhaust the $f$ sum rule in the $k \rightarrow 0$ limit, and multiexcitations. We emphasize that all the semiphenomenological theories of quantum fluids, e.g., quantum hydrodynamics, Landau's Fermi liquid theory, and extensions thereof, are essentially single-excitation theories. Because of this fact, these theories pioneered by Landau are simple, germane, and extremely useful.

As a rule the single-excitation theories give correctly the leading $k$ dependence of the structure functions. The exceptions are the structure functions $S_{l}(\mathbf{k})$ with $l \geq 3$, for which high-energy multiexcitation states are given considerable weight and thus may contribute to the leading $k$ dependence. When $l<3$ the single-excitation structure function $S_{l}^{(1)}(\mathbf{k})$ breaks down two orders in $k$ later due to multiexcitations, i.e., at the $O\left(k^{2}\right)$ term in the expression

$$
S_{l}(\mathbf{k}) \doteq S_{l}^{(1)}(\mathbf{k})\left[1+O\left(k^{2}\right)\right], \quad l=-1,0,2
$$

Since the single-excitation theories are well known, we will spend the remainder of the discussion on the general features of the effects of multi- 
excitations, in particular on some speculations concerning the structure functions and the discrete single-excitation spectrum.

\subsection{Generality of Backflow and Background}

Our treatment of $S(\mathbf{k}, \omega)$ was not tied to the presence or absence of long-range order. It is obvious, however, that the single-excitation spectrum $\omega_{k}$ can depend strongly on long-range order. For example, a crystalline lattice would introduce the periodicity of the reciprocal lattice onto the one-phonon spectrum $\omega_{\mathbf{k}}$ as a function of $\mathbf{k}$, solely from the kinematic constraints of energy and crystal momentum conservation. It is clear that such kinematic constraints cannot specify completely multiphonon processes and thus the multiphonon contributions do not follow the periodicity of the reciprocal lattice. For this reason, we expect that the backflow contribution to $S(\mathbf{k}, \omega)$ is relatively insensitive to the presence or absence of long-range order.

The backflow contribution to $S(\mathbf{k}, \omega)$ can be isolated by forming the ratio of the $\omega$ moment of $Z(\mathbf{k}) \delta\left(\omega-\omega_{k}\right)$ to the $f$ sum rule :

$$
\begin{aligned}
H_{1}(\mathbf{k}) & \equiv \int_{0}^{\infty} d \omega \omega Z(\mathbf{k}) \delta\left(\omega-\omega_{k}\right) / \int_{0}^{\infty} d \omega \omega S(\mathbf{k}, \omega) \\
& =1-2 m X_{1}(\mathbf{k}) / k^{2}
\end{aligned}
$$

Since $H_{1}(\mathbf{k})$ depends only on multiexcitations through the backflow $X_{1}(\mathbf{k})$, we expect that (48) to be relatively insensitive to long-range order. In particular, we can expect $H_{1}(\mathbf{k})$ to be roughly the same in the liquid and solid phases of ${ }^{4} \mathrm{He}$. Comparison of the solid ${ }^{4} \mathrm{He}$ data ${ }^{12}$ with the superfluid ${ }^{4} \mathrm{He}$ data ${ }^{7}$ confirms these theoretical expectations. Such a comparison was carried out by Werthamer ${ }^{13}$ but the one-phonon intensity function $Z(\mathbf{k})$ was incorrectly identified with the Debye-Waller factor, which as we have emphasized in Section 2.6, is not connected with backflow at all. This misinterpretation has spread to the extent that $Z(\mathbf{k})$ in superfluid ${ }^{4} \mathrm{He}$ has been called an effective Debye-Waller factor for the liquid. ${ }^{8}$

To appreciate the role of $e^{-2 W}$, we introduce the singular $f$ sum rule, which is a mathematical construct that gives the exact contribution to the $\omega$ moment of $S(\mathbf{k}, \omega)$ of all singular diagrams, i.e., those with an isolated one-phonon line, of the density response function. The singular dynamic structure function $S_{\text {sing }}(\mathbf{k}, \omega)$, which is proportional to the imaginary part of the singular diagrams, picks up, among other things, a delta function from the isolated one-phonon line. Note, however, that only one term in $S_{\text {sing }}(\mathbf{k}, \omega)$ is proportional to the one-phonon delta function; therefore it is 
erroneous to identify the whole of $S_{\text {sing }}(\mathbf{k}, \omega)$ with $Z(\mathbf{k}) \delta\left(\omega-\omega_{k}\right)$. Explicitly the singular $f$ sum rule for phonons in a solid ${ }^{14}$ is

$$
\int_{0}^{\infty} d \omega \omega S_{\text {sing }}(\mathbf{k}, \omega)=\{\exp [-2 W(\mathbf{k})]\} k^{2} / 2 m
$$

and for a Bose liquid ${ }^{10}$ is

$$
\int_{0}^{\infty} d \omega \omega S_{\text {sing }}(\mathbf{k}, \omega)=n_{0}\left[k^{2} / 2 m+M_{11}^{\mathrm{HF}}(\mathbf{k})-M_{12}^{\mathrm{HF}}(\mathbf{k})-\mu\right]
$$

where $n_{0}$ is the condensate density, $\mu$ is the chemical potential, and $M^{\mathrm{HF}}(\mathbf{k})$ is the Hartree-Fock matrix self-energy. In a solid $e^{-2 W}$ plays the role of $n_{0}$, in furnishing the link between the density correlation function and the fundamental amplitude correlation function. In other words if $e^{-2 w}\left(n_{0}\right)$ vanishes in a solid (Bose liquid), the poles of the density correlation function need not be the same as the displacement (field) amplitude correlation function. The interpretation of $e^{-2 W}$ as the one-phonon intensity function is equivalent to the identification of $n_{0}$ with $Z(\mathbf{k})$, which is clearly wrong:

From the present point of view, the anomaly in the solid bce ${ }^{4} \mathrm{He}$ data $^{12}$ appears to be in the lowest transverse mode $T_{1}[011]$, which does not show the general backflow but behaves as the harmonic one-phonon approximation (46).

It is also clear from the same reasoning that the background $X(\mathbf{k}, \omega)$ should be relatively insensitive to long-range order. Such a theoretical expectation is qualitatively confirmed in two separate regions in $(\mathbf{k}, \omega)$ space. At the high- $\omega$ and relatively low- $k$ region, light scattering measurements ${ }^{15}$ of solid hcp, solid bcc, and liquid ${ }^{3} \mathrm{He}$ showed very little change in the scattering profiles as a function of energy shift. At the high- $\omega$ and high- $k$ region where the single-excitation state $|1\rangle$ no longer exists, we find from the $f$ sum rule that the average multiexcitation energy is simply

$$
\bar{\omega}_{n 0}=k^{2} / 2 m X_{0}(\mathbf{k})
$$

which approaches the free-particle spectrum as $k \rightarrow \infty$. The background $X(\mathbf{k}, \omega)$ itself can be obtained by approximating the sum over $|n\rangle$ by the two-excitation state $|2\rangle$,

$$
X(\mathbf{k}, \omega) \approx \sum_{\mathbf{q}}\left|\left(\rho_{\mathbf{k}}^{+}\right)_{20}\right|^{2} \delta\left(\omega-k^{2} / 2 m-\mathbf{k} \cdot \mathbf{q} / m\right)
$$

which has a width (from the $\mathbf{k} \cdot \mathbf{q} / m$ term) that increases linearly with $k$. Neutron scattering measurements ${ }^{7.8 .12}$ have confirmed the existence of this "independent-particle" background in the various phases of ${ }^{4} \mathrm{He}$ : solid hcp, solid bcc, superfluid, and normal liquid: 


\subsection{Inflection Point in $S(k)$}

One of the results for neutral quantum fluids in Section 2 is that in the long-wavelength limit the backflow contribution to $S(\mathbf{k}, \omega)$ dominates the background contribution. Explicitly we have found that $Y(\mathbf{k}) \sim k^{3}$ and $X_{l}(\mathbf{k}) \sim k^{4}$ as $k \rightarrow 0$. Since $Y(\mathbf{k})$ is negative and $X_{l}(\mathbf{k})$ is positive, we expect from (11) that at long wavelengths (assuming a nominally linear phonon spectrum) $S(\mathbf{k})$ would show an inflection point due to the interplay between the negative backflow contribution and the positive continuum contribution. Note that this is a general property of the multiexcitation contributions to $S(\mathbf{k})$ and does not depend on the underlying particle statistics. However, this inflection property does depend on whether the interaction is Coulombic or not. In a charged quantum fluid, we have found that $S(\mathbf{k}) \sim k^{2}, Y(\mathbf{k}) \sim k^{4}$, $X_{l}(\mathbf{k}) \sim k^{4}$ as $k \rightarrow 0$. Hence to leading orders in $k$ we do not expect from these considerations an inflection point in $S(\mathbf{k})$ of a charged quantum fluid.

The location and visibility of the inflection point in $S(\mathbf{k})$ of a neutral quantum fluid depend of course on the relative magnitudes of the coefficients, which we now estimate for liquid ${ }^{4} \mathrm{He}$ and liquid ${ }^{3} \mathrm{He}$. If we write $Y(\mathbf{k})=-a k^{3}+\cdots, X_{0}=b k^{4}+\cdots$, and ignore the phonon dispersion, then the inflection point would occur at $k_{i}=a / 2 b$. From (13), we see that the ratio of the backflow to background contribution is

$$
-Y(\mathbf{k}) / X_{0}(\mathbf{k})=X_{1}(\mathbf{k})\left[\omega_{k} X_{0}(\mathbf{k})\right]^{-1}
$$

The right-hand side of (53) is just the ratio of the mean multiexcitation energy $\bar{\omega}_{n 0}$ to the single-excitation energy $\omega_{k}$. For liquid ${ }^{4} \mathrm{He}, \bar{\omega}_{n 0}$ is approximately ${ }^{7} 18 \mathrm{~K}$ and $\omega_{k}=c k$ in the long-wavelength region, where $c$ is the sound speed. Taking the long-wavelength limit, we find $a / b=\bar{\omega}_{n 0} / c$ $\approx 0.99 \AA^{-1}$ and the inflection point in $S(\mathbf{k})$ of ${ }^{4} \mathrm{He}$ should be at

$$
k_{i}^{(4)} \approx 0.5 \AA^{-1},
$$

which is in agreement with previous estimates. ${ }^{4}$ For liquid ${ }^{3} \mathrm{He}$, the situation is not so clear, since there are no measurements of the multiexcitations. If we make the assumption that $\bar{\omega}_{n 0}$ in ${ }^{3} \mathrm{He}$ is roughly the same as that in ${ }^{4} \mathrm{He}$, since the multiexcitations are more insensitive to the particle statistics than the single excitations, then from the sound speed ratio $c_{3} / c_{4} \approx 0.8$, we expect that the inflection point in $S(\mathbf{k})$ of ${ }^{3} \mathrm{He}$ should be at

$$
k_{i}^{(3)} \approx 0.6 \AA^{-1}
$$

Hallock ${ }^{16}$ has measured $S(\mathbf{k})$ for liquid ${ }^{4} \mathrm{He}$ and for liquid ${ }^{3} \mathrm{He}$ and has found a gentle shoulder displaying an inflection point in $S(\mathbf{k})$ for each of the quantum liquids. The positions of the inflection points are consistent with the above estimates. 


\subsection{Phonon Spectrum in Liquid ${ }^{3} \mathrm{He}$}

The phonon (zero-sound) spectrum in liquid ${ }^{3} \mathrm{He}$ is given by (25) or (26). Since liquid ${ }^{3} \mathrm{He}$ is a strong-coupling Fermi liquid in the sense that as $k \rightarrow 0$ the phonon (zero-sound mode) itself nearly exhausts the $f$ sum rule, we make the reasonable assumption that the phonon would dominate the $f$ sum rule over the finite range in $k$ in which it is well defined. In other words in (25) and (26), we neglect $X_{0}^{\mathrm{P}}$ and $X_{1}^{\mathrm{P}}$ so that $\omega_{k}^{\mathrm{L}} \rightarrow \omega_{k}^{\mathrm{F}}$ and obtain

$$
\omega_{k}=\omega_{k}^{F}-\left[X_{0}(\mathbf{k}) / S(\mathbf{k})\right]\left[\bar{\omega}_{n 0}-\omega_{k}\right]
$$

which shows that the effect of the multiexcitations is to push $\omega_{k}^{\mathrm{F}}$ downward. The effect of the neglected single pairs would be to push $\omega_{k}^{\mathrm{F}}$ upward. A rough plot of $\omega_{k}^{\mathrm{F}}$ for liquid ${ }^{3} \mathrm{He}$ shows a roton minimum at $k_{3}^{\mathrm{F}} \approx 1.7 \AA^{-1}$ with energy $\Delta_{3}^{\mathrm{F}} \approx 22 \mathrm{~K}$ and a maximum at $\sim 1.4 \AA^{-1}$ with energy $\sim 24 \mathrm{~K}$, and the whole curve $\omega_{k}^{\mathrm{F}}$ lies above the single-pair continuum. The impreciseness of these numbers is due to a lack of agreement of $S(\mathbf{k})$ as measured by $\mathrm{x}$-ray scattering. ${ }^{16,17}$ To investigate $\omega_{k}$, we form the ratio of (54) with the phonon spectrum of liquid ${ }^{4} \mathrm{He},(14)$,

$$
\frac{\omega_{k}^{(3)}}{\omega_{k}^{(4)}}=\frac{S^{(4)}(\mathbf{k})-X_{0}^{(4)}(\mathbf{k})}{S^{(3)}(\mathbf{k})-X_{0}^{(3)}(\mathbf{k})} \frac{k^{2} / 2 m_{3}-X_{1}^{(3)}(\mathbf{k})}{k^{2} / 2 m_{4}-X_{1}^{(4)}(\mathbf{k})}
$$

Since the small- $k$ behavior of $\omega_{k}^{(3)}$ is well known, we concentrate on the large- $k$ behavior. A very crude estimate can be obtained from (55) by extrapolating to the finite- $k$ region the multiexcitation equalities valid in the infinite- $k$ limit, viz., $X_{0}^{(3)}(\mathbf{k})=X_{0}^{(4)}(\mathbf{k})$ and $H_{1}^{(3)}(\mathbf{k})=H_{1}^{(4)}(\mathbf{k})$, where $H_{1}(\mathbf{k})$ is defined in (48). Equation (55) then reduces to

$$
\frac{\omega_{k}^{(3)}}{\omega_{k}^{(4)}} \approx \frac{m_{4}}{m_{3}} \frac{1-X_{0}(\mathbf{k}) / S(\mathbf{k})}{R(\mathbf{k})-X_{0}(\mathbf{k}) / S(\mathbf{k})}
$$

where $R(\mathbf{k}) \equiv S^{(3)}(\mathbf{k}) / S^{(4)}(\mathbf{k})$ and $X_{0}(\mathbf{k}) / S(\mathbf{k})$ refers to ${ }^{4} \mathrm{He}$. Using existing data ${ }^{7.16 .17}$ on ${ }^{3} \mathrm{He}$ and ${ }^{4} \mathrm{He}$, we estimate from (56) a minimum in $\omega_{k}^{(3)}$ at $k_{3} \approx 1.6 \AA^{-1}$ with energy $\Delta_{3} \approx 9 \mathrm{~K}$, if the phonon in ${ }^{3} \mathrm{He}$ is still well defined.

\subsection{Phonon Spectrum in ${ }^{3} \mathrm{He}-{ }^{4} \mathrm{He}$ Solutions}

The phonon spectrum in ${ }^{3}{ }^{3} \mathrm{He}-{ }^{4} \mathrm{He}$ solution can be written

$$
\omega_{k}=\frac{k^{2} / 2 \mu-X_{1}(\mathbf{k})}{S(\mathbf{k})-X_{0}(\mathbf{k})}-\frac{X_{0}^{\mathrm{P}}(\mathbf{k})}{S(\mathbf{k})-X_{0}(\mathbf{k})}\left[\bar{\omega}^{\mathrm{P}}-\omega_{k}\right]
$$

where $\mu$ is defined in (29) and $\bar{\omega}^{\mathrm{P}} \equiv X_{1}^{\mathrm{P}}(\mathbf{k}) / X_{0}^{\mathrm{P}}(\mathbf{k})$ is the average single-pair spectrum. At a low concentration $x$ of ${ }^{3} \mathrm{He}$ atoms dissolved into liquid 
${ }^{4} \mathrm{He}, \omega_{k}$ is approximately equal to the boson spectrum $\omega_{k}^{\mathrm{b}}$

$$
\omega_{k}^{\mathrm{b}}=\frac{k^{2} / 2 m_{\mathrm{b}}-X_{1}^{\mathrm{b}}(\mathbf{k})}{S^{\mathrm{b}}(\mathbf{k})-X_{0}^{\mathrm{b}}(\mathbf{k})}
$$

evaluated at the reduced solution density. Since a low concentration of ${ }^{3} \mathrm{He}$ in liquid ${ }^{4} \mathrm{He}$ forms a weak-coupling Fermi liquid, it is reasonable to assume that the leading $x$ correction to $\omega_{k}^{\mathrm{b}}$ comes from the single-pair states rather than the multiexcitations; then we have from (57)-(58) to leading order in $x$

$$
\omega_{k}-\omega_{k}^{\mathrm{b}} \approx \frac{X_{0}^{\mathrm{P}}(\mathbf{k})}{Z^{\mathrm{b}}(\mathbf{k})}\left(\omega_{k}^{\mathrm{b}}-\bar{\omega}^{\mathrm{P}}\right)+x \frac{k^{2}}{2 m_{\mathrm{f}} Z^{\mathrm{b}}(\mathbf{k})}+\cdots
$$

where the Bose intensity function $Z^{\mathrm{b}}(\mathbf{k}) \equiv S^{\mathrm{b}}(\mathbf{k})-X_{0}^{\mathrm{b}}(\mathbf{k})$ is known to be positive. At low $k, \bar{\omega}^{\mathrm{P}}<\omega_{k}^{\mathrm{b}}$ and the spectrum $\omega_{k}$ is repelled to an energy higher than $\omega_{k}^{\mathrm{b}}$, i.e., the leading $x$ correction increases the sound speed evaluated at the reduced solution density. If at large $k, \bar{\omega}^{\mathrm{P}}$ is larger (smaller) than $\omega_{k}^{\mathrm{b}}$, then the spectrum $\omega_{k}$ is repelled to an energy lower (higher) than $\omega_{k}^{\mathrm{b}}$. Note that the argument rests entirely on the form of the average singlepair spectrum $\bar{\omega}^{\mathrm{P}}$, which is not known. Nevertheless we see from (59) that the behavior of $\omega_{k}$ would reflect the qualitative features of $\bar{\omega}^{\mathrm{P}}$ as to whether $\bar{\omega}^{\mathrm{P}}$ crosses $\omega_{k}^{\mathrm{b}}$ or not. A similar conclusion was drawn on the basis of a detailed calculation ${ }^{18}$ using an extended quantum hydrodynamic model. The phonon shift $\omega_{k}-\omega_{k}^{\mathrm{b}}$ measured by neutron scattering ${ }^{19}$ is an oscillating function of $k$ with a minimum shift that is negative. Since the measurement was taken at $1.6 \mathrm{~K}$, which is much greater than the characteristic (Fermi) temperature ( $T_{\mathrm{F}} \approx 0.3 \mathrm{~K}$ ), the $T=0$ relation $(59)$ is not directly applicable. Nevertheless it is difficult to understand the negative shift on the basis of (59) unless $\bar{\omega}^{\mathrm{P}}$ crosses $\omega_{k}^{\mathrm{b}}$. A more definitive conclusion for $\bar{\omega}^{\mathrm{P}}$ awaits a low-temperature neutron scattering experiment.

\subsection{Concluding Remarks}

By the use of the $f$ sum rule, we have attempted to isolate the multiexcitation contributions to $S(\mathbf{k}, \omega)$, to separate it into backflow and background, and to illustrate its generality. We have seen how multiexcitations can affect the intensity function $Z(\mathbf{k})$ through the backflow, as well as give rise to a background $X(\mathbf{k}, \omega)$. The exact expression for backflow hopefully has clarified the concept of backflow pioneered by FC. From the exact expressions for the single-excitation spectrum $\omega_{k}$, we have seen how the multiexcitations effectively repel the single excitations, i.e., level repulsion. In short, the presence of multiexcitations is seen to have a profound effect on the single excitations. 
We have centered our attention on the $f$ sum rule because of its relationship to number conservation. The other sum rules involving $S(\mathbf{k}, \omega)$, which have not been used here, undoubtedly would be quite useful in setting bounds for various physical quantities (see, e.g., Ref. 6) and in determining the density excitation spectrum at long wavelengths (see, e.g., Ref. 20). Such considerations are important and are reserved for future study.

\section{APPENDIX. THE LEGGETT INEQUALITIES}

We give here a plain derivation of the Leggett ${ }^{11}$ inequalities for the Fermi liquid in a quantum solution, which were originally derived by fieldtheoretic techniques. The basic idea is to exploit the fact that in a quantum solution the fermion number density and spin density are conserved but in general not the current or spin current. We begin with the response functions

$$
\mathscr{F}_{\xi}(\mathbf{k}, \omega)=\sum_{v}\left|\left(\xi_{k}^{+}\right)_{v 0}\right|^{2} \frac{2 \omega_{v 0}}{\omega^{2}-\omega_{v 0}+i 0^{+}}
$$

where $\xi_{k}^{+}$can be the density, spin-density, current, or spin-current operator. The excited states $|v\rangle$, coupled to the ground state $|0\rangle$ by $\xi_{k}^{+}$, can be divided into the single $\xi$-excitation states $|1\rangle$ and the multiexcitation states $|n\rangle$. Concentrating on the longitudinal-current response function $\mathscr{F}_{j}$, we separate out the single-excitation part

$$
\begin{aligned}
& \mathscr{F}_{j}(\mathbf{k}, \omega)=\mathscr{\mathscr { F }}_{j}^{(1)}(\mathbf{k}, \omega)+\mathscr{F}_{j}^{(n)}(\mathbf{k}, \omega) \\
& \mathscr{F}_{j}^{(1)}(\mathbf{k}, \omega) \equiv \sum_{1}\left|\left(j_{k}^{+}\right)_{10}\right|^{2} \frac{2 \omega_{10}}{\omega^{2}-\omega_{10}^{2}+i 0+} \\
& \mathscr{F}_{j}^{(n)}(\mathbf{k}, \omega) \equiv \sum_{n}\left|\left(j_{k}^{+}\right)_{n 0}\right|^{2} \frac{2 \omega_{n 0}}{\omega^{2}-\omega_{n 0}^{2}+i 0+}
\end{aligned}
$$

Since the current is not conserved,

$$
\lim _{k / \omega \rightarrow 0} \mathscr{F}_{j}^{(1)}(\mathbf{k}, \omega)=0, \quad \lim _{k / \omega \rightarrow 0} \mathscr{F F}_{j}^{(n)}(\mathbf{k}, \omega) \leq 0
$$

From number conservation it follows that

$$
\frac{\omega^{2}}{k^{2}} \mathscr{F}_{\rho}(\mathbf{k}, \omega)=\frac{N}{m}+\mathscr{F}_{j}(\mathbf{k}, \omega)
$$

Using (A5), we see that

$$
\lim _{k / \omega \rightarrow 0} \frac{\omega^{2}}{k^{2}} \mathscr{F}(\mathbf{k}, \omega) \leq \frac{N}{m}
$$


It is well known ${ }^{1}$ that the left-hand side of (A7) is equal to $N\left(1+\frac{1}{3} F_{1}\right) / m^{*}$; hence we obtain one of Leggett's inequalities

$$
m^{*} /\left(1+\frac{1}{3} F_{1}\right) \geq m
$$

A similar analysis of the spin longitudinal-current response function yields

$$
m^{*} /\left[1+(1 / 12) Z_{1}\right] \geq m
$$

Equation (A8) is to be compared with (39).

\section{ACKNOWLEDGMENTS}

I would like to acknowledge many helpful conversations with D. L. Bartley, H. Gould, and L. M. Sander.

\section{REFERENCES}

1. D. Pines and P. Nozieres, Theory of Quantum Liquids (Benjamin, New York, 1966).

2. R. P. Feynman, Phys. Rev. 94, 267 (1954).

3. R. P. Feynman and M. Cohen, Phys. Rev. 102, 1189 (1956).

4. A. Miller, D. Pines, and P. Nozieres, Phys. Rev. 127, 1452 (1962).

5. D. Pines and C.-W. Woo, Phys. Rev. Letters 24, 1044 (1970).

6. D. Hall and E. Feenberg, Ann. Phys. (N.Y.) 63, 335 (1971).

7. R. A. Cowley and A. D. B. Woods, Can. J. Phys. 49, 177 (1971).

8. A. D. B. Woods and R. A. Cowley, Rep. Prog. Phys. 36, 1135 (1973), and references therein.

9. V. K. Wong and H. Gould, Phys. Lett. 39A, 331 (1972).

10. V. K. Wong and H. Gould, Ann. Phys. (N.Y.) 83, 252 (1974).

11. A. J. Leggett, Ann. Phys. (N.Y.) 46, 76 (1968).

12. V. J. Minkiewicz, T. A. Kitchens, G. Shirrane, and E. G. Osgood, Phys. Rev. A 8, 1513 (1973), and references therein.

13. N. R. Werthamer, Phys. Rev. Lett. 28. 1102 (1972),

14. V. Ambegaokar, J. M. Conway, and G. Baym, in Lattice Dynamics, R. F. Wallis, ed. (Pergamon, London, 1965), p. 261.

15. R. E. Slusher and C. M. Surko, Phys. Rev. Lett. 27, 1699 (1971).

16. R. B. Hallock, Phys. Rev. Lett. 26, 618 (1971); J. Low Temp. Phys. 9, 109 (1972), and references therein.

17. E. K. Achter and L. Meyer, Phys. Rev. 188, 291 (1969).

18. D. L. Bartley, V. K. Wong, and J. E. Robinson, J. Low Temp. Phys. 17, 551 (1974).

19. J. M. Rowe, D. L. Price, and G. E. Ostrowski, Phys. Rev. Lett. 31, 510 (1973).

20. S. Ichimaru and T. Tange, Phys. Rev. Lett. 32, 102 (1974). 